\title{
Attentional Modulation of Affective Versus Sensory Processing: Functional Connectivity and a Top-Down Biased Activation Theory
} of Selective Attention

\author{
Fabian Grabenhorst ${ }^{1}$ and Edmund T. Rolls ${ }^{2}$ \\ ${ }^{1}$ Department of Experimental Psychology, University of Oxford; and ${ }^{2}$ Oxford Centre for Computational Neuroscience, Oxford, \\ United Kingdom
}

Submitted 16 April 2010; accepted in final form 14 July 2010

\begin{abstract}
Grabenhorst F, Rolls ET. Attentional modulation of affective versus sensory processing: functional connectivity and a top-down biased activation theory of selective attention. J Neurophysiol 104: 1649-1660, 2010. First published July 14, 2010; doi:10.1152/jn.00352.2010. Topdown selective attention to the affective properties of taste stimuli increases activation to the taste stimuli in the orbitofrontal cortex (OFC) and pregenual cingulate cortex (PGC), and selective attention to the intensity of the stimuli increases the activation in the insular taste cortex, but the origin of the top-down attentional biases is not known. Using psychophysiological interaction connectivity analyses, we showed that in the anterior lateral prefrontal cortex (LPFC) at $\mathrm{Y}=$ $53 \mathrm{~mm}$ the correlation with activity in OFC and PGC seed regions was greater when attention was to pleasantness compared with when attention was to intensity. Conversely, we showed that in a more posterior region of the LPFC at $\mathrm{Y}=34$ the correlation with activity in the anterior insula seed region was greater when attention was to intensity compared with when attention was to pleasantness. We also showed that correlations between areas in these separate processing streams were dependent on selective attention to affective value versus physical intensity of the stimulus. We then propose a biased activation theory of selective attention to account for the findings and contrast this with a biased competition theory of selective attention.
\end{abstract}

\section{N T R O D U C T I O N}

Top-down selective attention has recently been shown to have remarkable effects on how the brain responds to taste and olfactory stimuli (Grabenhorst and Rolls 2008; Rolls et al. 2008a). Instructions to pay attention to and later rate the pleasantness of a taste increase the activations to taste measured with functional magnetic resonance imaging (fMRI) in the orbitofrontal cortex (OFC) and pregenual cingulate cortex (PGC) (Grabenhorst and Rolls 2008), where the subjective pleasantness of taste is represented (de Araujo et al. 2003b; Grabenhorst et al. 2008a, 2009; Kringelbach et al. 2003; Rolls and Grabenhorst 2008), but not the primary taste cortex in the anterior insula (Grabenhorst and Rolls 2008), where the subjective intensity and identity of taste are represented (de Araujo et al. 2003b; Grabenhorst et al. 2008a; Haase et al. 2009; Kringelbach et al. 2003; Rolls and Grabenhorst 2008; Small et al. 2003). Instructions to pay attention to and later rate the intensity of a taste increase the activations to taste in the insular taste cortex but not in the OFC and PGC (Grabenhorst and Rolls 2008). Similar effects have been demonstrated for olfactory stimuli, with selective attention to pleasantness increasing activations to an odor in the OFC and PGC, and attention to intensity

\footnotetext{
Address for reprint requests and other correspondence: E. T. Rolls, Oxford Centre for Computational Neuroscience, Oxford, UK (E-mail: Edmund.Rolls @ oxcns.org www.oxcns.org).
}

increasing activations in the pyriform cortex and olfactory tubercle but not in the OFC and PGC (Rolls et al. 2008a). Consistently, trying to detect the presence of taste in a tasteless solution enhances activity in the insular taste cortex and overlying operculum but not the OFC (Veldhuizen et al. 2007).

These findings have shown that when selective attention is directed to the affective properties of a sensory stimulus, such as its pleasantness, the brain systems engaged to represent the sensory stimulus are different from those engaged when attention is directed to the nonaffective physical properties of a stimulus such as its intensity. This differential biasing of brain regions engaged in processing a sensory stimulus, depending on whether the cognitive demand is for affect-related versus more sensory-related processing, may be an important aspect of how cognition and attention operate. Understanding these processes has many implications for understanding the effects not only of taste and olfactory, but also of other sensory stimuli and for understanding how attention can influence emotion (Rolls 2005; Rolls and Grabenhorst 2008) and food intake (Rolls, unpublished data) by selectively altering responses to affective, rewarding stimuli. Further, these findings have implications for understanding the neural processes involved in affective valuation and decision making, for they show that representations of expected value and the value of decision outcomes in the OFC and PGC (Behrens et al. 2008; Grabenhorst et al. 2008b; Kable and Glimcher 2007; Plassmann et al. 2007; Rolls and Grabenhorst 2008; Rolls et al. 2008b, 2010) can be influenced by cognition and attention.

Top-down selective attention involves a modulation, probably by biased competition, of the responses of neurons to incoming sensory stimuli (Deco and Rolls 2005a; Desimone and Duncan 1995; Rolls 2008b; Rolls and Deco 2002). Top-down biased competition involves an attentional signal from elsewhere in the brain, biasing some but not other neurons in a cortical area, so that when the bottom-up or forward input is received, the biased population in the cortical area wins the competition that is implemented using feedback inhibition through the local cortical inhibitory neurons that receive excitatory inputs from the pyramidal cells in the cortical area. Nonlinear effects in selective attention, often described as multiplicative, involve a much greater response to a stimulus when there is a top-down selective influence on the neurons that respond to the stimulus. Using integrate-and-fire neuronal network simulation analyses, it has been shown that the underlying mechanism can involve linear summation of the top-down attentional and bottom-up sensory input, combined with the threshold nonlinear- 
ity of the neurons when they are operating close to threshold on a nonlinear, then steeply rising part of their activation function (Deco and Rolls 2005a; Rolls 2008b).

In the present study, we investigated the source of the top-down attentional modulation. It is thought that this is often some part of the prefrontal cortex (Corbetta and Shulman 2002; Downar et al. 2000; Kanwisher and Wojciulik 2000), in which a short-term memory implemented by associatively modifiable recurrent collateral synapses can hold a set of neurons active for the attentional period, with the neurons representing the attentional task instruction or rule that is currently valid. This set of active neurons is the source of the top-down synaptic inputs to neurons in areas in which attention modulates neuronal activity to stimuli (Deco and Rolls 2005a; Rolls 2008b; Rolls and Deco 2002). Previous fMRI studies have shown that the lateral prefrontal cortex (LPFC) represents current task demands, such as what particular type of task will be performed on a given trial (Sakai and Passingham 2003, 2006). However, the source of top-down attentional modulation of affective representations in the OFC and PGC is unknown. Nor do we know whether the source for the top-down affective attentional modulation of responses to taste stimuli is the same as the source for the top-down attentional modulation of responses to the intensity of taste. Here, we investigated both these key issues using psychophysiological interaction (PPI) methodology (Friston et al. 1997).

Understanding the ways in which the pleasantness of the taste, or smell, or sight of food is encoded in the brain and can be affected by factors such as top-down cognition (de Araujo et al. 2005; Grabenhorst et al. 2008a) or attention as investigated here is important in the context of understanding how the brain controls food intake, body weight, and problems such as overeating and obesity. When a food is eaten to satiety its reward value or pleasantness is affected, and not its intensity, and the reduction in the reward value or pleasantness as represented in brain areas that represent the reward value of food is how food intake in controlled (Rolls 1981, 2005, 2007a; Rolls and Grabenhorst 2008; Rolls et al. 1983). The aim of this investigation was therefore to lead to a better understanding of how top-down attention influences food reward and pleasantness, and it is possible that control of attention to food could be a factor among many useful in controlling appetite (Rolls 2005, 2007b, 2010).

\section{METHODS \\ Overall design}

We used the identical taste stimulus [0.1 M monosodium glutamate (MSG) with $0.005 \mathrm{M}$ inosine monophosphate, referred to throughout this study for brevity as monosodium glutamate (see Araujo et al. 2003a)] in two different types of trials. A trial started $5 \mathrm{~s}$ before the taste delivery with the visual attentional instruction: either "Remember and Rate Pleasantness" or "Remember and Rate Intensity," which was shown until the end of the taste period. The $0.75 \mathrm{ml}$ taste stimulus was delivered at $t=$ $5 \mathrm{~s}$. The taste period was from $t=5 \mathrm{~s}$ until $t=14 \mathrm{~s}$ and in this period a red cross was also present, indicating that swallowing should not occur. The differences between the activations in this period were a measure of the top-down selective attention instructions while the taste was being delivered. [We note that to use top-down attention, one needs to hold the object of attention in mind, in this case pleasantness or intensity. This requires a short-term memory. Short-term memory is thus a sine qua non of selective attention (Rolls 2008b; Rolls and Deco 2002) and it is the source of this top-down bias from a short-term memory system in which we are interested in this investigation.] After the end of the taste period the visual instruction and red cross were turned off and a green cross was shown cueing the subject to swallow. After $2 \mathrm{~s}$ a tasteless rinse was delivered with a red cross, and the rinse period was from $t=16$ until $t=$ $23 \mathrm{~s}$, when the green cross appeared to cue a swallow. After this the rating of pleasantness or intensity was made using button-press operated visual analog rating scales ranging continuously from +2 (very pleasant) to -2 (very unpleasant) for pleasantness and 4 (intense) to 0 (very weak) for intensity, as previously described (Rolls et al. 2003b).

These two trial types, in which the instructions were to remember and rate either pleasantness or intensity, were those crucial to the present investigation and its hypotheses. They were interspersed in random permuted sequence with other trials that were part of a different investigation in which there was no pretrial instruction or attention period, and cognitive modulation by word labels of taste and flavor processing in the brain was being investigated (Grabenhorst et al. 2008a). The tastes used on these other trials were monosodium glutamate at concentrations of 0.1 and $0.4 \mathrm{M}$, the flavors were $0.1 \mathrm{M}$ monosodium glutamate and vegetable odor, and the word labels for the taste condition were "Rich and delicious taste" or "monosodium glutamate." Each of the seven trial types was presented in random permuted sequence nine times. This general protocol and design have been successfully used in previous studies to investigate taste cortical areas (de Araujo et al. 2003c; Grabenhorst et al. 2008a; McCabe and Rolls 2007; O'Doherty et al. 2001). Because seven trial types were being run in the scanner at the same time and included different stimuli (Grabenhorst et al. 2008a), and no instructions were given about the number of stimuli being used or that the stimuli were the same on the "Remember and Rate Intensity" and "Remember and Rate Pleasantness" trials, the participants simply had to concentrate on following the instructions about what aspect of the taste stimulusintensity or pleasantness - had to be rated on that trial.

In a previous analysis of this data set we focused on identifying the target areas where top-down attentional effects influence responses to taste stimuli (Grabenhorst and Rolls 2008). Here we investigate the source of the top-down attention signal using the PPI methodology (Friston et al. 1997).

\section{Participants}

Twelve healthy volunteers ( 6 male and 6 female, age range 21-35 yr) participated in the study. Ethical approval (Central Oxford Research Ethics Committee) and written informed consent from all subjects were obtained before the experiment. The subjects had not eaten for $3 \mathrm{~h}$ before the investigation. With our sample size of 12 participants, statistically significant effects in random-effects group analyses were found as described in the following text.

\section{Stimuli and stimulus delivery}

The taste stimulus was monosodium glutamate (0.1 M MSG and 0.005 $\mathrm{M}$ inosine monophosphate). We included a tasteless control solution containing the main ionic components of saliva $(25 \mathrm{mM} \mathrm{KCl}+2.5 \mathrm{mM}$ $\mathrm{NaHCO}_{3}$ ), which when subtracted from the effects produced by the taste stimulus allowed somatosensory and any mouth movement effects to be distinguished from the effects purely related to taste (de Araujo et al. 2003a; O'Doherty et al. 2001). This is an important control condition that we have pioneered to allow taste areas to be shown independently of any somatosensory effects produced by introducing a fluid into the mouth (de Araujo et al. 2003a,b; O'Doherty et al. 2001). For the PPI analyses described herein, explicit subtraction of effects related to the rinse was not applied because the rinse effect was common to each of the two attentional conditions involved in the analyses.

The stimuli were delivered to the subject's mouth through four Teflon tubes (one for each of the three taste or flavor stimuli and a separate tube for the tasteless rinse control) that were held between the 
lips. Each Teflon tube ( $\sim 3 \mathrm{~m}$ in length) was connected to a separate reservoir via a syringe and a one-way syringe activated check valve (Model 14044-5, World Precision Instruments), which allowed 0.75 $\mathrm{ml}$ of any stimulus to be delivered at the time indicated by the computer.

\section{fMRI data acquisition}

Images were acquired with a 3.0-T Varian/Siemens whole-body scanner at the Centre for Functional Magnetic Resonance Imaging at Oxford, where $27 \mathrm{~T} 2 *$-weighted echo-planar imaging (EPI) coronal slices with in-plane resolution of $3 \times 3 \mathrm{~mm}$ and between-plane spacing of $4 \mathrm{~mm}$ were acquired every $2 \mathrm{~s}$ (repetition time [TR] $=2$ ). We used the techniques that we have developed over a number of years (de Araujo et al. 2003a; O'Doherty et al. 2001) and, as described in detail by Wilson et al. (2002), we carefully selected the imaging parameters to minimize susceptibility and distortion artifact in the OFC. The relevant factors include imaging in the coronal plane, minimizing voxel size in the plane of the imaging, as high a gradient switching frequency as possible $(960 \mathrm{~Hz})$, a short echo time of $28 \mathrm{~ms}$, and local shimming for the inferior frontal area. The matrix size was $64 \times 64$ and the field of view was $192 \times 192 \mathrm{~mm}$. Continuous coverage was obtained from +62 (anteroposterior [AP]) to -46 (AP). A whole-brain T2*-weighted EPI volume of the above-cited dimensions and an anatomical T1 volume with coronal plane slice thickness $3 \mathrm{~mm}$ and in-plane resolution of $1 \times 1 \mathrm{~mm}$ were also acquired.

\section{fMRI data analysis}

The imaging data were analyzed using SPM5 (Statistical Parametric Mapping, Wellcome Trust Centre for Neuroimaging, London). Preprocessing of the data used SPM5 realignment, reslicing with sinc interpolation, normalization to the Montreal Neurological Institute coordinate system (Collins et al. 1994), and spatial smoothing with a $6 \mathrm{~mm}$ full width at half-maximum isotropic Gaussian kernel. Time series nonsphericity at each voxel was estimated and corrected for (Friston et al. 2002) and a high-pass filter with a cutoff period of 128 $\mathrm{s}$ was applied. To investigate task-dependent correlations between brain areas during the taste period a finite impulse response (FIR) analysis was performed as implemented in SPM, to make no assumption about the time course based on the temporal filtering property of the hemodynamic response function (Gottfried et al. 2006; Henson 2004; Yacubian et al. 2006). We used 16 delta functions in the FIR analysis, spaced at intervals of the TR $(2 \mathrm{~s})$ and starting $2 \mathrm{~s}$ before the onset of the visual cue at time $-5 \mathrm{~s}$, instructing the subject to "Remember and rate pleasantness" (or intensity) on that trial. This basis set individually considers each time bin after stimulus onset, to model the blood oxygen level dependent (BOLD) response, and can capture any possible shape of response function up to a given frequency limit. In this model, the parameter estimate for each time bin represents the average BOLD response at that time. These parameter estimates are directly proportional to the BOLD signal.

Data were analyzed statistically for each subject individually (firstlevel analysis) and for the group (second-level analysis). At the single-subject level, the parameter estimate maps for the FIR analysis for the time delays at which taste-related activations were expected were estimated (in our case, the FIR values for times starting 6 and 8 $\mathrm{s}$ after taste delivery were used and are consistent with the hemodynamic delays inherent in the BOLD response; Henson 2004). Following smoothness estimation (Kiebel et al. 1999), in the first stage of analysis condition-specific experimental effects (parameter estimates or regression coefficients pertaining to the effect size of the PPI) were obtained via the general linear model (GLM) in a voxelwise manner for each subject. The statistical parametric maps from each individual data set were then entered into second-level, random-effects analyses accounting for both scan-to-scan and subject-to-subject variability. More precisely, the sets of individual statistical maps corresponding to a specific effect of interest were entered as covariates in multiple regression models as implemented in SPM5 and the corresponding group effects were assessed by applying linear contrasts (again following smoothness estimation) to the (second-level) parameter estimates generating a $t$-statistics map for each group effect of interest. The SPM contrast analyses performed tested for a difference between paying attention to pleasantness versus intensity and vice versa. For the contrast analysis reported in Grabenhorst and Rolls (2008) the main regressors of interest in our GLM modeled the onset of the taste stimuli on the attention to pleasantness and attention to intensity trials for the FIR times corresponding to the taste period as described earlier, starting at 6 and $8 \mathrm{~s}$, using $t$-contrasts as implemented in SPM. This type of approach was chosen because the 6- and 8-s bins were identified (by examining average FIR BOLD response time courses in the present data set as well as in other data sets where taste-related responses were measured) as reflecting the peak of the taste-related BOLD response in the selective attention design in taste-related areas of interest including the insular taste cortex and OFC without also reflecting any effects related to the attention instructions at the start of the trial. Other task periods, including specifically the attentional instruction period, swallowing periods, rinse period, and rating periods, as well as other trial types not related to the present investigation were modeled as effects of no interest. The group-level $t$-tests were performed with 11 degrees of freedom.

We performed PPI analyses (Friston et al. 1997; Gitelman et al. 2003) to investigate how activity in pairs of brain regions is modulated as a function of task (attentional condition). The PPI models performed at the single-subject level included three regressors. The first regressor consisted of the time series of activity in a seed brain area identified in the contrast analyses. The time series was extracted in each subject by drawing a 6-mm sphere around the peak voxel from the group contrast analysis and then finding the individual's peak voxel within that sphere. The second regressor consisted of a taskrelated contrast (attention to pleasantness vs. attention to intensity or vice versa). The third regressor consisted of the interaction between the first and second regressors. The statistical parametric maps from each individual data set were then entered into second-level, randomeffects analyses. Only the statistical maps of the individual subjects relating to the interaction regressor were taken to the second (group) level of analysis. The a priori defined areas of interest for which we report results included brain areas where activations to taste stimuli have been found in previous studies, including the medial and lateral $\mathrm{OFC}$, the pregenual part of the cingulate cortex, and the taste and oral somatosensory parts of the insular cortex (de Araujo et al. 2003a,c; Grabenhorst et al. 2008a; McCabe and Rolls 2007; Nitschke et al. 2006; O'Doherty et al. 2001; Schoenfeld et al. 2004) and areas of the LPFC where activations related to task set, attentional instructions, and remembering rules that guide task performance have been found, including specifically parts of the middle and inferior frontal gyrus (Beck and Kastner 2009; Bengtsson et al. 2009; Deco and Rolls 2005a; Kouneiher et al. 2009; Rossi et al. 2009; Sakai and Passingham 2003, 2006; Veldhuizen et al. 2007).

We applied small volume (false discovery rate) corrections for multiple comparisons for which $P<0.05$ (although the exact corrected probability values are provided) (Genovese et al. 2002), with a radius corresponding to the full width at half-maximum of the spatial smoothing filter used. Peaks are reported for which $P<0.05$ corrected for false discovery rate, although the actual corrected probability values are given in the text. For effects outside our a priori regions of interest we applied a statistical threshold of $P<0.05$, corrected for false discovery across the whole brain. In addition to the statistical criterion just described for a significant effect calculated for the peak voxel of a region of activation in an a priori defined region based on earlier findings, we used the additional statistical test (see Gottfried et al. 2002; O'Doherty et al. 2003, 2006) that the results reported were significant in global contrast and/or correlation analyses 
using significant using the criterion of $P<0.001$, uncorrected for multiple comparisons, and these additional statistics confirmed the same effects in the a priori regions in all cases in this study unless otherwise stated.

For voxels where the correlations between the percentage blood oxygen level dependent (\% BOLD) values in two brain regions were significantly task dependent (i.e., where there were significant PPI effects with $P<0.05$ using small volume correction), we produced graphs to show how the $\%$ BOLD signal of the two regions was related as a function of attentional task. These were produced for each subject by taking the average of the BOLD response from both brain regions in the two time bins at 6 and $8 \mathrm{~s}$ after the delivery of the taste stimulus, on each trial. For each individual subject we navigated to the nearest peak under the relevant PPI effect that lay within a 6-mm sphere of the group peak. For each subject the means were calculated in discretized ranges of the range of \% BOLD values (e.g., -2 to $-1.75,-1.75$ to -1.5 , etc.) and then these values were averaged across subjects. The \% BOLD signal change was obtained by plotting the event-related responses in SPM5, which calculates the \% BOLD signal change relative to the grand mean over all voxels in a given session.

\section{RES U L T S}

As we have reported previously (Grabenhorst and Rolls 2008), a contrast of trials where attention was being paid to taste pleasantness with trials where attention was to intensity revealed significant effects in the OFC [ $\left[\begin{array}{lll}-6 & 14 & -20\end{array}\right]$ and a region to which it projects, the pregenual cingulate cortex $[-4$ $46-8]$. The reverse contrast of trials where attention was to intensity versus trials where attention was to pleasantness revealed significant effects in the right anterior insular taste cortex [42 $18-14$ ] and the right mid insular cortex [40 -2 4]. To investigate task-dependent functional connectivity of these areas with other brain regions we performed PPI analyses using the above-cited brain areas as seed regions. The location of the seed regions is indicated in Fig. $1 B$.

\section{Lateral prefrontal cortex}

TASK-DEPENDENT CORRELATIONS WITH OFC AND PGC. PPI analyses identified two areas of the anterior LPFC where functional connectivity with the OFC and PGC was modulated as a function of the attentional condition. In one area of the anterior LPFC the correlation with activity in the OFC seed region was greater when attention was to pleasantness compared with when attention was to intensity [-40 54 14], $z=3.32, P=0.029$ (Fig. 2A). In another area of the anterior LPFC the correlation with activity in the PGC seed region was greater when attention was to pleasantness compared with when attention was to intensity [ $-4250-2]$, $z=3.44, P=0.017$ (Fig. $2 A$ ). The parameter estimates for the PPI analyses at these sites are shown in Fig. $2 B$.

To illustrate how the correlation between $\mathrm{OFC}$ and anterior LPFC is modulated as a function of attentional condition, Fig. $2 C$ shows the \% BOLD signal in the anterior LPFC as a function of the \% BOLD signal in the OFC, with the data plotted separately for the attention to pleasantness condition and the attention to intensity condition.

TASK-DEPENDENT CORRELATIONS WITH ANTERIOR INSULA AND MID INSULA. PPI analyses identified two areas of the more posterior LPFC where functional connectivity with the anterior and mid insula was modulated as a function of the attentional condition. In one area of the LPFC the correlation with activity in the anterior insula seed region was greater when attention was to intensity compared with when attention was to pleasantness [-38 34 14], $z=2.87, P<0.05$ (Fig. 3A). (We note that although the effect shown in Table 1 between the anterior insula and this part of the LPFC is contralateral, the activations on the two sides of the anterior insula are in any case correlated, especially when attention is being paid to intensity, as shown in Table 1.) The peak coordinate in the anterior-posterior dimension of this intensity-related effect in the LPFC (Y = 34.17 $\pm 0.90 \mathrm{SE})$ was significantly different from and more posterior than the corresponding pleasantness-related effect described earlier, where correlated activity with the OFC was

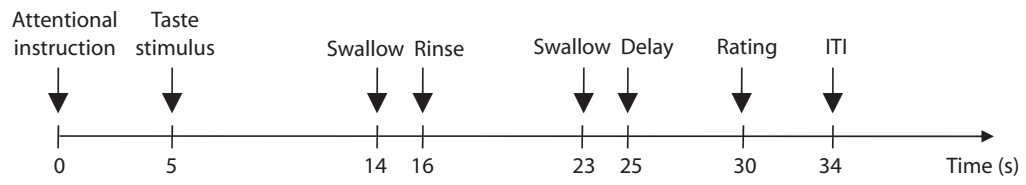

B Seed regions

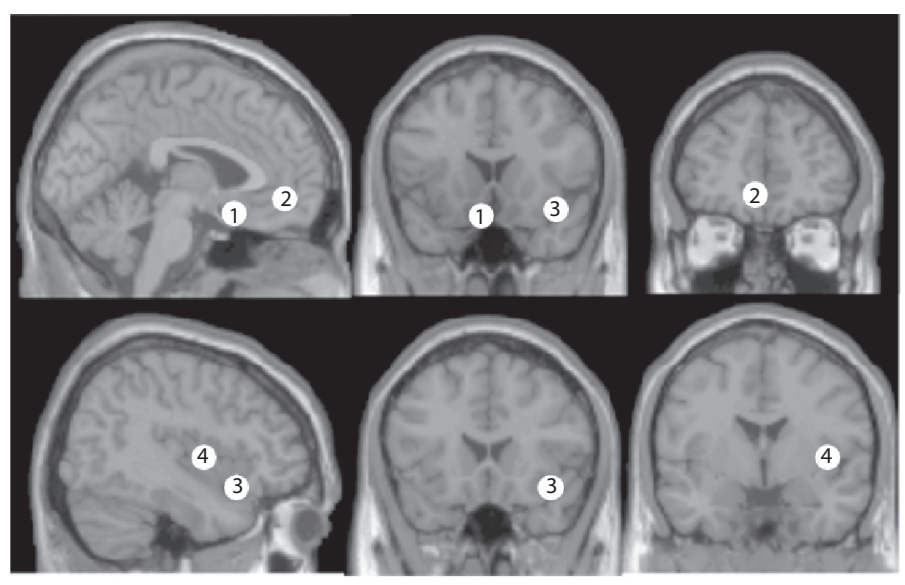

FIG. 1. A: the time course of a trial. The taste stimulus and the rinse were delivered at the times shown. ITI, start of the intertrial interval. $B$ : the seed regions for the psychophysiological interaction (PPI) analyses. The orbitofrontal cortex (OFC, (1) and pregenual cingulate cortex (PGC, (2) were seed regions at which attention to the pleasantness of a taste modulated the response to a taste. The anterior insula (3) and mid insula (4) were seed regions at which attention to the intensity of a taste modulated the response to a taste. 

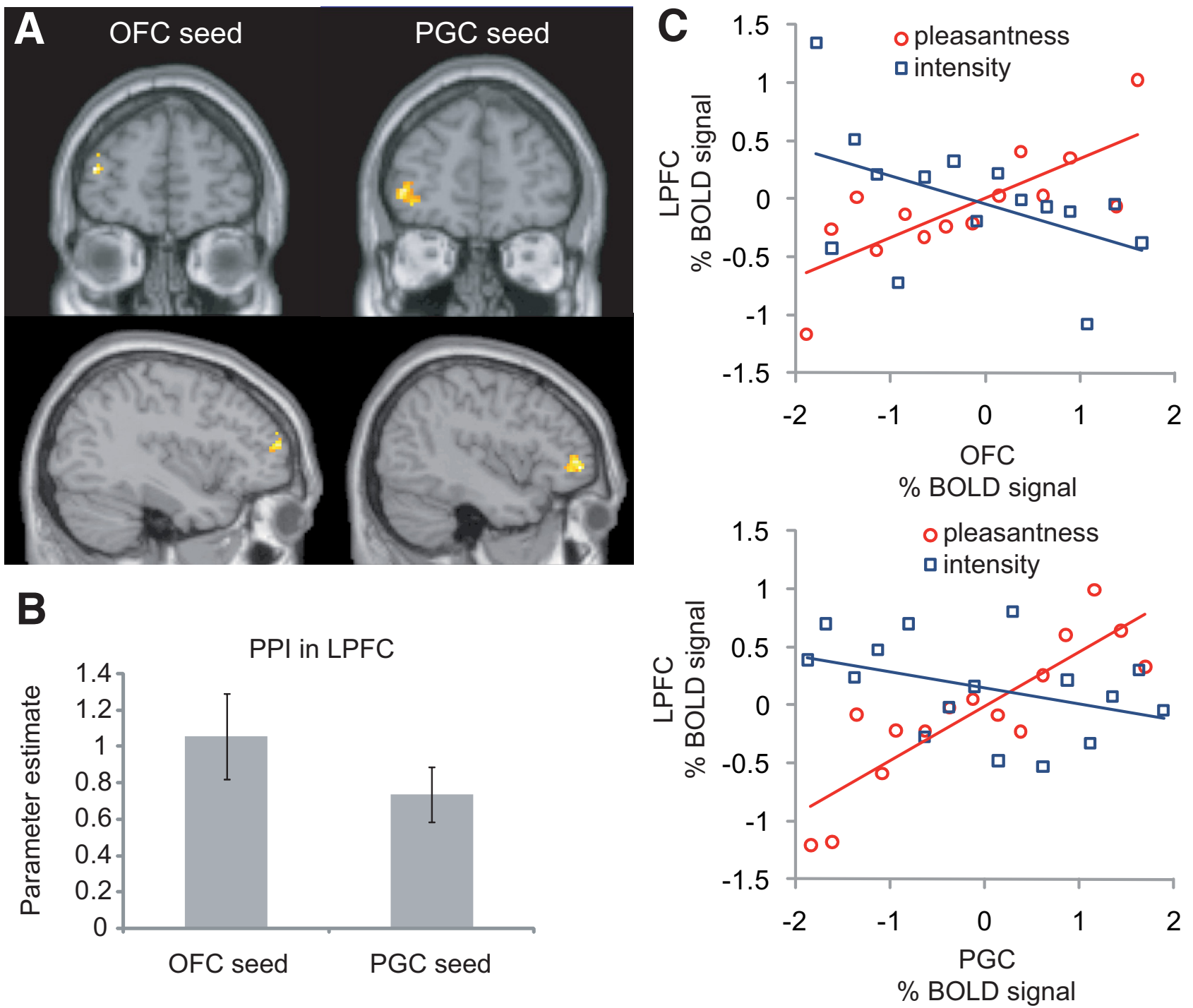

FIG. 2. Regions of anterior lateral prefrontal cortex (LPFC) at which the correlation with activity in OFC and PGC seed regions was greater when attention

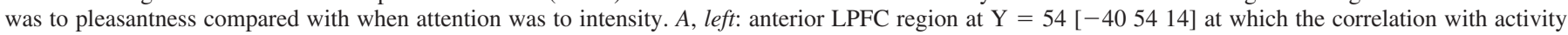

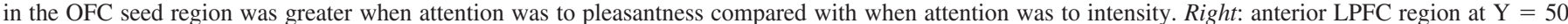

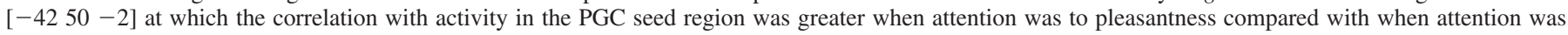

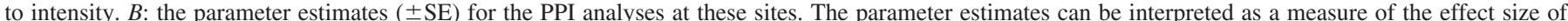

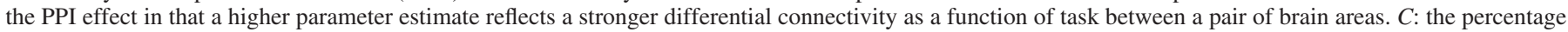

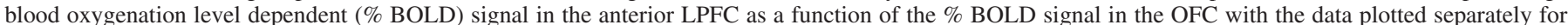
the attention to pleasantness condition and the attention to intensity condition.

found $\left(\mathrm{Y}=53.3 \pm 0.96 \mathrm{SE} ; t_{11}=14.34, P=1.8 \times 10^{-8}\right)$. This indicates that different parts of the LPFC are involved in attention to pleasantness versus attention to intensity.

We further investigated whether the task-related connectivity between the LPFC and anterior insula on the one hand and LPFC and OFC on the other is related to the same underlying mechanism of top-down attentional modulation. We performed a statistical test to compare the slopes of the regression lines describing the relationship of the BOLD signals in these pairs of brain regions during the attention to intensity and attention to pleasantness trials (the blue regression line in Fig. $3 C$ and the red regression line in Fig. $2 C$ ). The two slopes were statistically indistinguishable $\left(t_{23}=0.41, P>0.5\right)$ in this comparison with 12 participants, providing an indication that the mechanism underlying the task-dependent functional connectivity between these pairs of brain regions is qualitatively similar.
In another area of the LPFC the correlation with activity in the mid insula seed region was greater when attention was to intensity compared with when attention was to pleasantness [-46 34 0], $z=3.32, P=0.014$ (Fig. 3A). The parameter estimates for the PPI analyses at these sites are shown in Fig. $3 B$.

To illustrate how the correlation between anterior insula and LPFC is modulated as a function of attentional condition, Fig. $3 C$ shows the \% BOLD signal in the LPFC as a function of the $\%$ BOLD signal in the anterior insula with the data plotted separately for the attention to pleasantness condition and the attention to intensity condition.

\section{Task-dependent correlations between the OFC and the PGC}

Functional connectivity between different areas of the OFC and the PGC was modulated as a function of attentional 

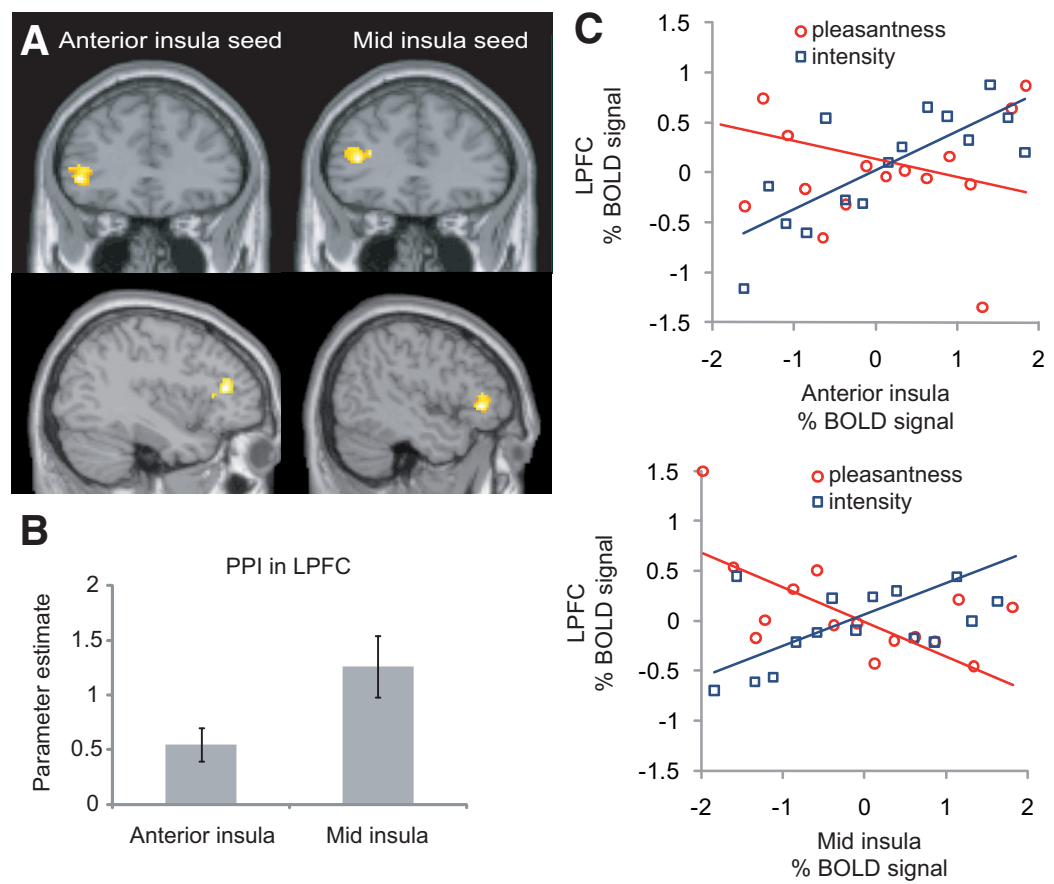

FIG. 3. Regions of LPFC at which the correlation with activity in insular cortex seed regions was greater when attention was to intensity compared with when attention was to pleasantness. A, left: LPFC region at $\mathrm{Y}=34\left[\begin{array}{lll}-38 & 34 & 14\end{array}\right]$ at which the correlation with activity in the anterior insula seed region was greater when attention was to intensity compared with when attention was to pleasantness. Right: LPFC region at $\mathrm{Y}=34[-46340]$ at which the correlation with activity in the mid insula seed region was greater when attention was to intensity compared with when attention was to pleasantness. $B$ : the parameter estimates for the PPI analyses at these sites. $C$ : the \% BOLD signal in the LPFC as a function of the \% BOLD signal in the anterior insula with the data plotted separately for the attention to pleasantness condition and the attention to intensity condition.

condition. In the PGC the correlation with activity in the OFC seed region was greater when attention was to pleasantness compared with when attention was to intensity [ -14562$], z=$ 3.26, $P<0.013$ and [ -4368 ] $, z=2.76, P<0.05$ (Fig. 4A). The extent of this effect in the PGC overlapped with the PGC seed region. In the anterior medial OFC the correlation with activity in the PGC seed region was greater when attention was to pleasantness compared with when attention was to intensity [-8 $56-14], z=3.99, P=0.002$ (Fig. 4A). This OFC area was relatively more anterior than the OFC seed area and corresponds to a location where correlations with subjective pleasantness ratings of different types of reward have been found in previous studies (de Araujo et al. 2003c; Grabenhorst and Rolls 2009; Grabenhorst et al. 2007). The parameter estimates for the PPI analyses at these sites are shown in Fig. $4 B$.

\section{Task-dependent correlations between different areas of the insula}

Functional connectivity between different areas of the insula was modulated as a function of attentional condition. In the left anterior insula the correlation with activity in the right anterior

TABLE 1. Brain regions where significant effects in psychophysiological interaction analyses were found

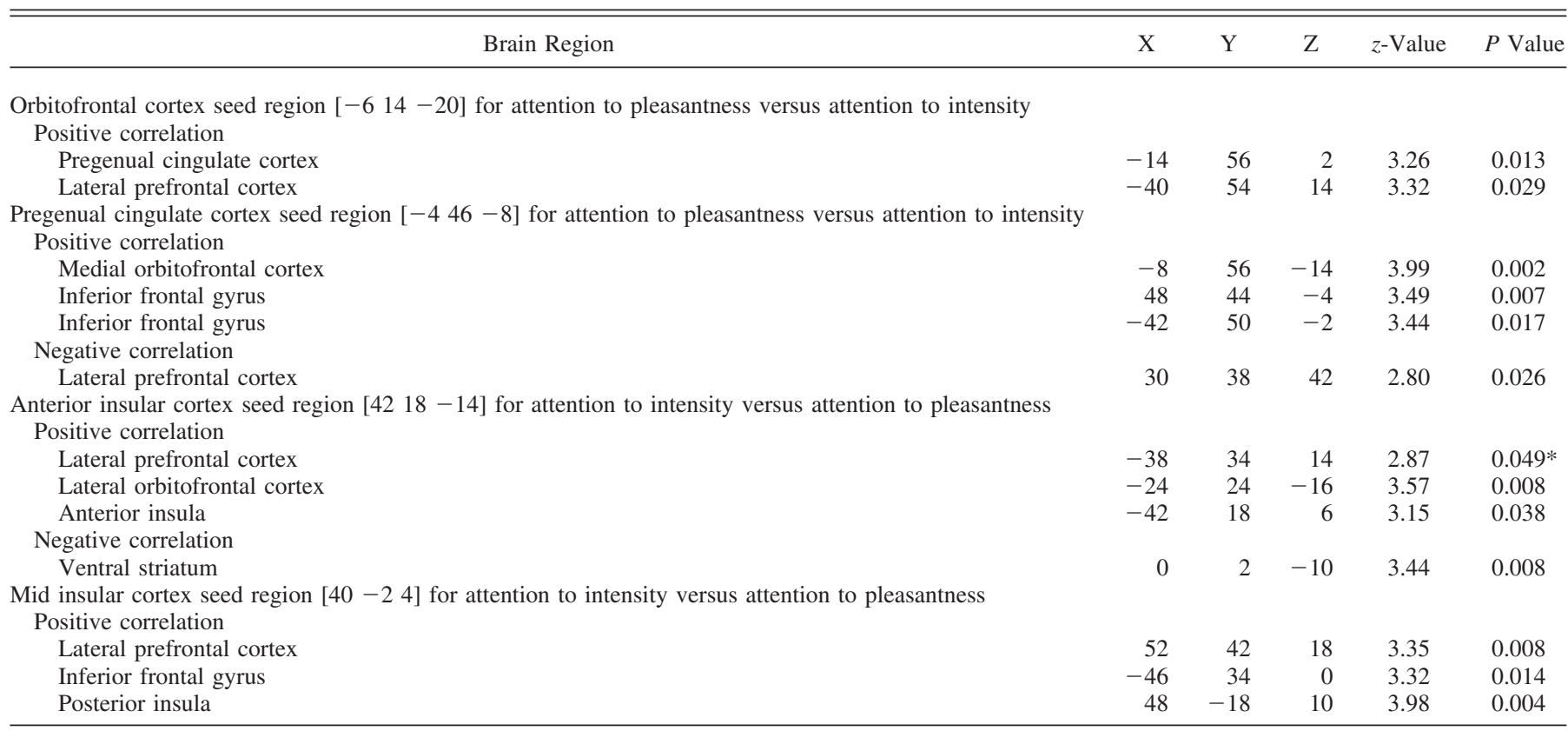

All effects are significant at $P<0.05$ (small volume correction) and $P<0.001$ (uncorrected in whole brain analyses), except where otherwise stated. $* P<$ 0.05 (small volume correction) and $P=0.002$ (uncorrected in whole brain analysis). 

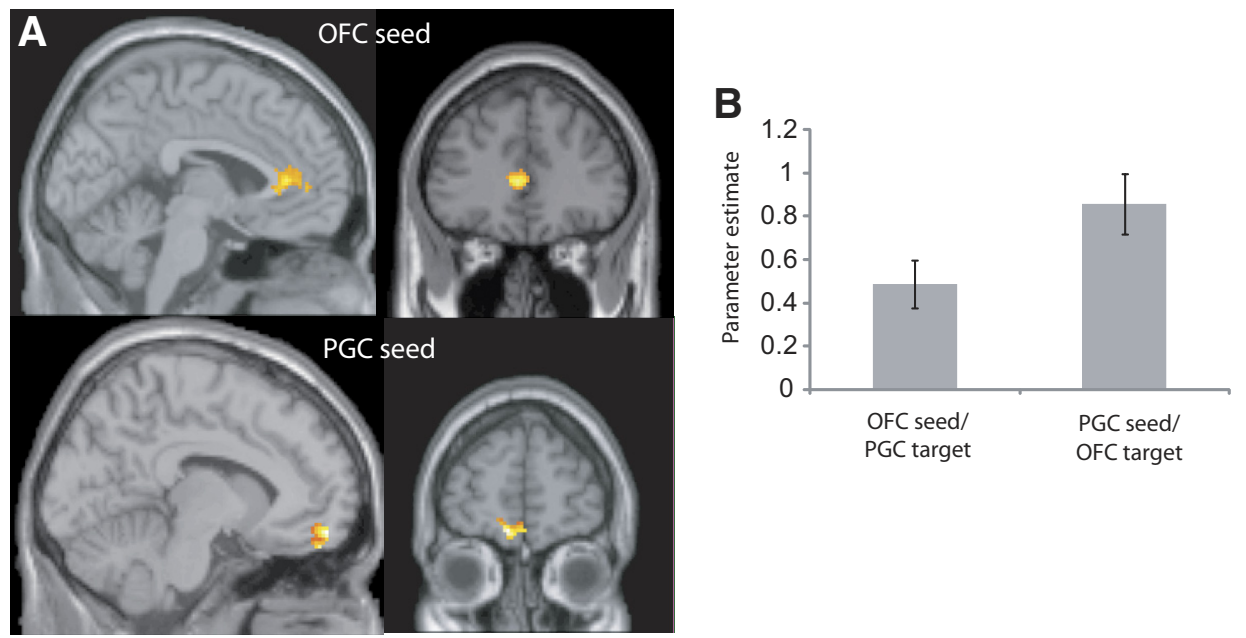

FIG. 4. Task-dependent correlations between the OFC and PGC. A, top: OFC seed. In the PGC ([- $\left.\begin{array}{lll}-14 & 56 & 2\end{array}\right]$ and $\left.\left[\begin{array}{lll}-4 & 36 & 8\end{array}\right]\right)$ the correlation with activity in the OFC seed region was greater when attention was to pleasantness compared with when attention was to intensity. Bottom: PGC seed. In the anterior medial OFC ([-8 $56-14])$ the correlation with activity in the PGC seed region was greater when attention was to pleasantness compared with when attention was to intensity. $B$ : the parameter estimates (mean $\pm \mathrm{SE}$ ) for the PPI analyses at these sites.

insula was greater when attention was to intensity compared with when attention was to pleasantness $[-42186], z=3.15$, $P=0.038$ (Fig. 5A). In a posterior region of the insula the correlation with activity in the mid insula seed region was greater when attention was to intensity compared with when attention was to pleasantness [48 - 1412 ], $z=3.98, P=0.004$ (Fig. 5B). The parameter estimates for the PPI analyses at these sites are shown in Fig. $5 B$.

\section{I S C U S S I O N}

We know of no previous investigations of the source of the top-down bias for attention in the taste system. There is also very little previous evidence on the top-down source of the bias when attention is to affective (emotional) versus sensory aspects (e.g., the intensity) of the same stimulus (Pessoa 2009). In this investigation we found that two sites where selective attention to pleasantness increased the activation to taste, the OFC and a region to which it is connected, the PGC, both had functional connectivity with a quite anterior (mean $\mathrm{Y} \approx 50$ ) part of the LPFC (see Fig. 2 and Table 1). These parts of the OFC and PGC are a functionally appropriate target site for a top-down attentional modulation, in that their activations are correlated with the subjectively rated pleasantness of the taste (Grabenhorst and Rolls 2008). Moreover, the LPFC has been shown to represent current task sets and attentional demands for different types of tasks (Sakai and Passingham 2003, 2006). We note that the statistics used in the calculation of PPI effects (Friston et al. 1997) do not reveal the directionality of the connectivity. However, given that the prefrontal cortex has a powerfully developed recurrent collateral system that provides the basis for the short-term memory (Deco and Rolls 2005a; Rolls 2008b; Rolls and Deco 2002) that is needed to hold the subject of attention active, providing the source of the bias for top-down biased competition (Deco and Rolls 2005a; Desimone and Duncan 1995; Rolls 2008b; Rolls and Deco 2002), that prefrontal cortex lesions impair attention (Beck and Kastner 2009; Rossi et al. 2009), and that activations in areas of the LPFC are related to task set, attentional instructions, and remembering rules that guide task performance (Beck and Kastner 2009; Bengtsson et al. 2009; Deco and Rolls 2005a; Kouneiher et al. 2009; Rossi et al. 2009; Sakai and Passingham 2003, 2006; Veldhuizen et al. 2007), the directionality in this case is likely to be from the prefrontal cortex to the OFC and PGC. Consistently, direct anatomical connections exist between the LPFC and the OFC and PGC (Price 2006).

The conclusion that these findings suggest is therefore that a part of the LPFC, not a site normally implicated in affective value and emotion, may be able to modulate emotion-/affectrelated processing in the brain by a top-down attentional influence. This may be one way in which higher cognitive functions, such as a reasoning-based strategy and route to
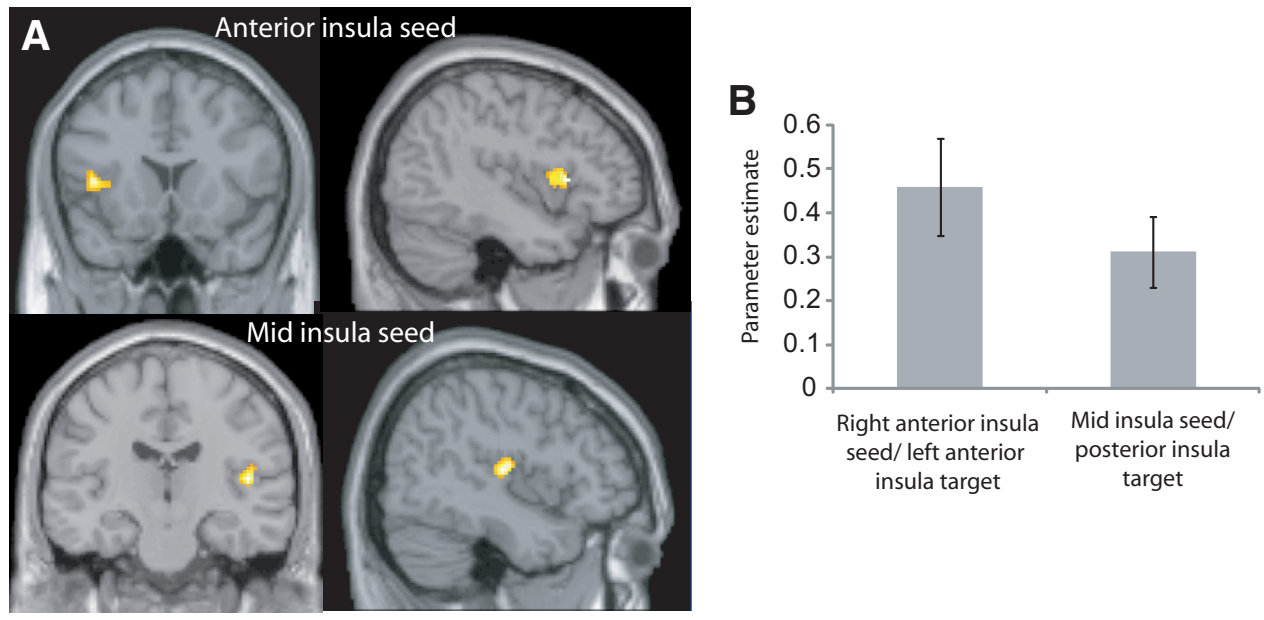

FIG. 5. Task-dependent correlations within the insular cortex. A, top: anterior insular cortex seed. In the left anterior insular cortex ([ -428 6]) the correlation with activity in the right anterior insular cortex seed region was greater when attention was to intensity compared with when attention was to pleasantness. Bottom: mid insular cortex seed. In the posterior insular cortex ([48 - 14 12]) the correlation with activity in the mid insular cortex seed region was greater when attention was to intensity compared with when attention was to pleasantness. $B$ : the parameter estimates (mean $\pm \mathrm{SE}$ ) for the PPI analyses at these sites. 
action, or verbal instruction to direct processing toward or away from emotion-related brain processing, or conscious volition, can influence the degree to which the affect-related parts of the brain process incoming (or potentially remembered) stimuli that can produce emotional responses. This is thus a part of the way in which cognition can influence and control emotion (Pessoa 2009; Rolls 2005; Rolls, unpublished data).

We also found that two sites where selective attention to intensity increased the activation to the taste delivery into the mouth, the anterior and mid insula, both had functional connectivity with a less anterior (mean $\mathrm{Y} \approx 37$ ) part of the LPFC (see Fig. 2 and Table 1). These parts of the insula are a functionally appropriate site for a top-down attentional modulation, in that their activations are correlated with the subjectively rated intensity of the taste (Grabenhorst and Rolls 2008; Grabenhorst et al. 2008a). The anterior insular site may be the primary taste cortex (de Araujo and Rolls 2004; de Araujo et al. 2003a; Pritchard et al. 1986; Rolls 2008a; Yaxley et al. 1990) and the mid-insular site a region activated by other oral including somatosensory and fat texture inputs, from the oral cavity (de Araujo and Rolls 2004; de Araujo et al. 2003b) and perhaps by taste per se (Small et al. 2003), in that the activations there were correlated with the trial-by-trial subjective ratings of the taste intensity made during the scanning (Grabenhorst and Rolls 2008). In the analyses described here, such somatosensory inputs could contribute to the attention-dependent correlations found between the mid insula and other areas.

The interpretation of this functional connectivity revealed with PPI (Friston et al. 1997) is that the prefrontal cortex and orbitofrontal/pregenual cingulate areas covary in their activations more strongly when attention is directed to pleasantness than to intensity. In this study, the implication is that when the activity in the orbitofrontal and pregenual cingulate areas is high, as it is on trials when attention is paid to pleasantness relative to trials when attention is paid to intensity, then activations in this prefrontal cortex region are also high. A large source of this variation that gives rise to the PPI effect is thus the difference in the activations on different trial types, which can be captured by the mean activations of both sites (OFC/PGC and prefrontal cortex) on each of the two trial types. However, in addition to this source of variation, it could be that when two areas are functionally interacting strongly, there may be an additional contribution to the connectivity term produced by the trial-by-trial variation within a type of trial. For example, on trials on which pleasantness is the subject of attention, then any small variation on a particular trial in the prefrontal cortex would be expected to be reflected in the activations in the OFC/PGC. This effect would arise because when both areas are active, the neurons in each area may be operating on a relatively linear part of their activation function, producing strong coupling, whereas when one or both areas are relatively inactive, with only spontaneous firing, then the neurons may be subject to some effects produced by being close to the firing threshold, such that small changes in input may produce a smaller than linear effect on the output. This trial-by-trial variation corresponds in information theoretic analysis of neuronal covariation to a "noise" effect compared with a "signal" effect (Oram et al. 1998; Rolls 2008b; Rolls et al. 2003a).
The way that we think of top-down biased competition as operating normally in, for example, visual selective attention (Desimone and Duncan 1995) is that within an area (e.g., a cortical region), some neurons receive a weak top-down input that increases their response to the bottom-up stimuli (Desimone and Duncan 1995), potentially supralinearly if the bottom-up stimuli are weak (Deco and Rolls 2005a; Rolls 2008b; Rolls and Deco 2002). The enhanced firing of the biased neurons then, via the local inhibitory neurons, inhibits the other neurons in the local area from responding to the bottom-up stimuli. This is a local mechanism, in that the inhibition in the neocortex is primarily local, being implemented by cortical inhibitory neurons that typically have inputs and outputs over no more than a few millimeters (Douglas et al. 2004; Rolls 2008b; Rolls and Deco 2002). This model of biased competition is illustrated in Fig. 6B. The locally implemented biased competition situation may not apply in the present case, where we have facilitation of processing in a whole cortical area (e.g., OFC or PGC) or even cortical processing stream (e.g., the linked OFC and PGC), in which any taste neurons may reflect pleasantness and not intensity. So the attentional effect might more accurately be described in this case as biased activation, without local competition being part of the effect. This biased activation theory and model of attention, illustrated in Fig. 6A, is a rather different way to implement attention in the brain than biased competition and each mechanism may apply in different cases, or both mechanisms in some cases.

The outputs of the separate processing streams showing biased activation (Fig. 6A) may need to be compared later to lead to a single behavior. One way in which this comparison could take place is by both outputs entering a single network cortical attractor model of decision making, in which positive feedback implemented by the excitatory recurrent collateral connections leads through nonlinear dynamics to a single winner, which is ensured by competition between the different possible attractor states produced through inhibitory neurons (Deco and Rolls 2006; Rolls and Deco 2010; Wang 2002, 2008). A second way in which the competition could be implemented is by that usually conceptualized as important in biased competition (Deco and Rolls 2005a,b; Desimone and Duncan 1995; Rolls and Deco 2002), in which a feedforward competitive network using inhibition through local inhibitory neurons provides a way for a weak top-down signal to bias the output, especially if the bottom-up inputs are weak (Deco and Rolls 2005b; Rolls 2008b; Rolls and Deco 2002) and this implementation is what is shown at the bottom of Fig. $6 \mathrm{~B}$. A third way in which the biased activation reflected in the output of the streams shown in Fig. $6 \mathrm{~A}$ could be taken into account is by a mechanism such as that in the basal ganglia, where in the striatum the different excitatory inputs activate $\gamma$-aminobutyric acid (GABA) neurons, which then directly inhibit each other to make the selection (Rolls 2005, 2008b).

The difference between biased competition and biased activation may be especially important in the context of functional neuroimaging because biased activation, in which processing in whole cortical areas is facilitated by selective attention, can be revealed by functional neuroimaging, which operates at relatively low spatial resolution, in the order of millimeters. In contrast, biased competition may selectively facilitate some pyramidal neurons within a local cortical area, which then through the local GABA inhibitory neurons compete with the 
A

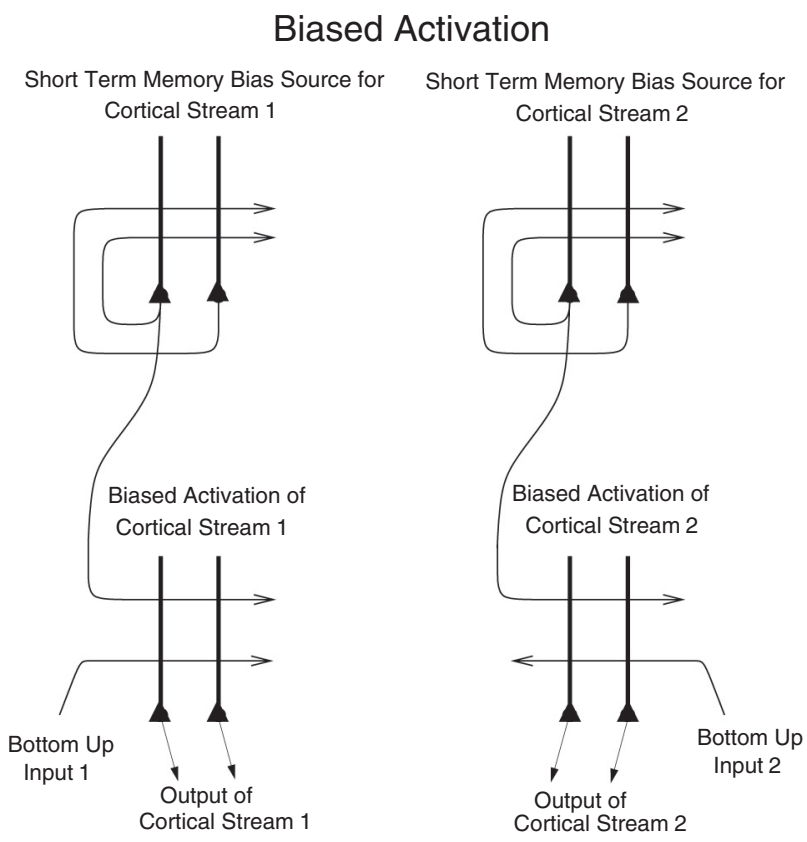

\section{B}

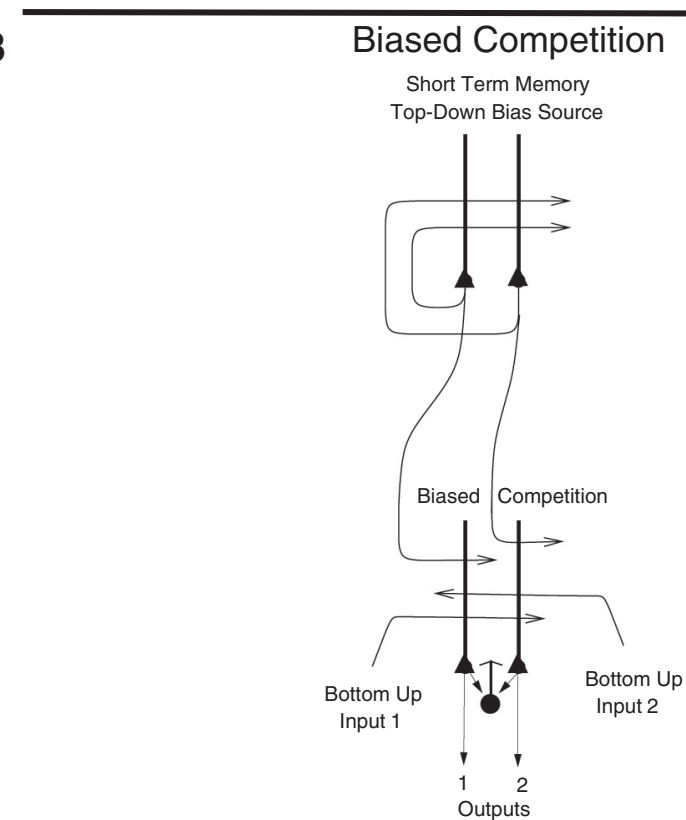

other pyramidal neurons in the area receiving bottom-up input. In this situation, in which some but not other neurons within a cortical area are showing enhanced firing, functional neuroimaging may not be able to show which local population of pyramidal cells is winning the competition due to the top-down bias. The evidence presented in this investigation is that not only the processing streams, but also even the short-term memory systems in the prefrontal cortex that provide the top-down source of the biased activation are physically separate, as illustrated in Fig. $6 \mathrm{~A}$.

A possibility arising from this model is that some competition may occur somewhere in the attentional system before the output stage and one possible area is within the prefrontal cortex, where it is a possibility that the attractors that implement the short-term memory for attention to pleasantness (at $\mathrm{Y} \approx 50$ ) may inhibit the attractors that implement the short-
FIG. 6. A: biased activation. The short-term memory systems that provide the source of the top-down activations may be separate (as shown) or could be a single network with different attractor states for the different selective attention conditions. The top-down short-term memory systems hold what is being paid attention to active by continuing firing in an attractor state and bias separately either cortical processing system 1 or cortical processing system 2 . This weak top-down bias interacts with the bottom-up input to the cortical stream and produces an increase of activity that can be supralinear (Deco and Rolls $2005 \mathrm{~b}$ ). Thus the selective activation of separate cortical processing streams can occur. In the example, stream 1 might process the affective value of a stimulus and stream 2 might process the intensity and physical properties of the stimulus. The outputs of these separate processing streams then must enter a competition system, which could be for example a cortical attractor decision-making network that makes choices between the two streams, with the choice biased by the activations in the separate streams (see text). $B$ : biased competition. There is usually a single attractor network that can enter different attractor states to provide the source of the top-down bias (as shown). If it is a single network, there can be competition within the short-term memory attractor states, implemented through the local $\gamma$-aminobutyric acid (GABA) inhibitory neurons. The top-down continuing firing of one of the attractor states then biases in a top-down process some of the neurons in a cortical area to respond more to one than the other of the bottom-up inputs, with competition implemented through the GABA inhibitory neurons (symbolized by a filled circle), which make feedback inhibitory connections onto the pyramidal cells (symbolized by a triangle) in the cortical area. The thick vertical lines above the pyramidal cells are the dendrites. The axons are shown with thin lines and the excitatory connections by arrowheads. term memory for attention to intensity (at $\mathrm{Y} \approx 37$ ), which could occur if there is some physical overlap between their zones of activation, even if the peaks are well separated. Some evidence for this possibility was found in the present investigation, in that the correlation between the \% BOLD activations in these two prefrontal cortex regions was $r=-0.72(P=$ $0.0034)$ on the pleasantness trials and $r=-0.8(P<0.001)$ on the intensity trials. In a biased competition model (Fig. $6 B$ ) we would normally think of the short-term memory attractors that provide the source of the bias as being within the same single attractor network, so that there would be competition between the two attractor states through the local inhibitory interneurons. In the biased activation model (Fig. 6A), it is an open issue about whether the attractors that provide the source of the top-down bias are in the same single network or are physically separate, making interactions between the attractor states dif- 
ficult through the short-range cortical inhibitory neurons. The findings just described indicate that in the case of top-down control of affective versus intensity processing of taste stimuli, although the two attractors are somewhat apart in the prefrontal cortex, there is some functional inhibitory interaction between them.

The principle of biased activation providing a mechanism for selective attention probably extends beyond processing in the affective versus sensory coding cortical streams. It may provide the mechanism also for effects in, for example, the dorsal versus the ventral visual system, in which attention to the motion of a moving object may enhance processing in the dorsal stream and attention to the identity of the moving object may enhance processing in the ventral visual stream (Brown 2009). Similar biased activation may contribute to the different localization in the prefrontal cortex of systems involved in "what" versus "where" working memory (Deco et al. 2004). Biased activation as a mechanism for top-down selective attention may be widespread in the brain and may be engaged when there is segregated processing of different attributes of stimuli.

In summary, the two mechanisms for top-down attention can be distinguished as follows. If the top-down signals operate to influence competition within an area implemented through a set of local inhibitory interneurons, this is biased competition (Fig. 6B). If whole systems (e.g., the OFC and PGC) have their activity increased relative to that in separate brain areas and systems, then we would term this biased activation. So the criterion for a difference is in terms of whether there is a local effect of the top-down modulation within an area or whether whole systems are influenced by the top-down signal before the outputs of the separate streams (Fig. 6A) may interact to produce an output. Further imaging and other studies will be able to show whether biased activation applies in other brain systems. For example, biased activation (Fig. 6A) is predicted to apply in systems where different attributes of the same stimulus are processed in separate cortical areas and streams. A good example is the ventral versus dorsal visual streams, in which a model described by Deco and Rolls predicts that 1 ) activation in a whole series of dorsal stream areas including parietal cortex and MT will be higher when attention is directed to the spatial location of the stimulus and 2) activation in a whole series of ventral stream areas including the inferior temporal visual cortex and V4 will be higher when attention is being paid to the identity of the stimulus (Deco and Lee 2004; Deco and Rolls 2004; Rolls 2008b; Rolls and Deco 2002). In contrast, biased competition (Fig. $6 B$ ) is predicted to apply where the processing of different attributes of a stimulus is being processed in the same cortical area by a single interconnected population of neurons. Examples occur in the visual system (e.g., V2 and V4) where attention can modulate the responses of a single neuron to different stimuli both of which fall within its receptive field (Deco and Rolls 2005b; Rolls 2008b). In this case, the responses of a single neuron are biased by top-down attention to respond differently to two stimuli within its receptive field and differential activation of different cortical processing streams is not predicted to be found with fMRI. Of course competition at the neuronal level will be found at some stage of processing with biased activation processing of attention, at a stage where the cortical streams 1 and 2 of Fig. $6 A$ do finally come together before behavior is produced. We note that the top-down source of the attentional bias could be from a single network, or from different networks, for both types of attentional system. However, separate cortical short-term memories for each attentional state are more likely where biased activation applies because separate cortical areas tend to receive connections from different parts of the prefrontal cortex (Jones and Powell 1970). That is what was found to apply in the present research investigation.

Some of the PPI effects shown in Table 1 are of further interest. The functional connectivity between the OFC and a region to which it is connected, the PGC, during attention to pleasantness versus intensity supports the hypothesis that these are part of an affect processing stream (Rolls 2005; Rolls and Grabenhorst 2008) through which processing is facilitated by attention to pleasantness/affective value. Consistently, a recent study found correlations between activity in different parts within the OFC during a pleasantness evaluation task when a taste was present compared with when no taste was present (Bender et al. 2009). The effects of PPI to contralateral areas such as between the two anterior insular regions indicate either cross-coupling or a common source of (probably top-down attentional) input during attention to intensity. Increased functional connectivity between the mid and posterior insular regions could reflect a neural system underlying the evaluation of what type of stimulus is in the mouth and what its physical properties are (de Araujo and Rolls 2004; de Araujo et al. 2003b; Veldhuizen et al. 2007).

In conclusion, we have shown here using psychophysiological interaction functional connectivity analyses that there are separate prefrontal cortex sites that could provide the origin of the top-down bias for selective attention to the affective value versus the intensity of a stimulus, a taste. We have also shown that functional connectivity within the affective value system for taste including the orbitofrontal and pregenual cingulate cortices is increased during selective attention to affective value versus intensity, and that functional connectivity within parts of the insula is increased during selective attention to intensity versus affective value. We have also proposed a biased activation theory of selective attention to account for the results and compared this with a biased competition theory of selective attention.

\section{A C K N OW LE D G MENTS}

We thank Dr. T. C. Nichols of The University of Warwick Applied Functional Neuroimaging Group and Department of Statistics for advice on the psychophysiological interaction analyses.

\section{G R A N T S}

This work was supported by grants from the Gottlieb-Daimler- and Karl Benz-Foundation, and the Oxford Centre for Computational Neuroscience to F. Grabenhorst. The investigation was performed at the Centre for Functional Magnetic Resonance Imaging of the Brain at Oxford University.

\section{I S C L OS URES}

No conflicts of interest, financial or otherwise, are declared by the author(s).

\section{REFERENCES}

Beck DM, Kastner S. Top-down and bottom-up mechanisms in biasing competition in the human brain. Vision Res 49: 1154-1165, 2009. 
Behrens TE, Hunt LT, Woolrich MW, Rushworth MF. Associative learning of social value. Nature 456: 245-249, 2008.

Bender G, Veldhuizen MG, Meltzer JA, Gitelman DR, Small DM. Neural correlates of evaluative compared with passive tasting. Eur J Neurosci 30: 327-338, 2009.

Bengtsson SL, Haynes JD, Sakai K, Buckley MJ, Passingham RE. The representation of abstract task rules in the human prefrontal cortex. Cereb Cortex 19: 1929-1936, 2009.

Brown JM. Visual streams and shifting attention. Prog Brain Res 176: 47-63, 2009

Collins DL, Neelin P, Peters TM, Evans AC. Automatic 3D intersubject registration of MR volumetric data in standardized Talairach space. $J$ Comput Assist Tomogr 18: 192-205, 1994.

Corbetta M, Shulman GL. Control of goal-directed and stimulus-driven attention in the brain. Nat Rev 3: 201-215, 2002.

de Araujo IET, Kringelbach ML, Rolls ET, Hobden P. The representation of umami taste in the human brain. J Neurophysiol 90: 313-319, 2003a.

de Araujo IET, Kringelbach ML, Rolls ET, McGlone F. Human cortical responses to water in the mouth, and the effects of thirst. J Neurophysiol 90: 1865-1876, 2003b.

de Araujo IET, Rolls ET. The representation in the human brain of food texture and oral fat. J Neurosci 24: 3086-3093, 2004.

de Araujo IET, Rolls ET, Kringelbach ML, McGlone F, Phillips N. Taste-olfactory convergence, and the representation of the pleasantness of flavour, in the human brain. Eur J Neurosci 18: 2059-2068, 2003 c.

de Araujo IET, Rolls ET, Velazco MI, Margot C, Cayeux I. Cognitive modulation of olfactory processing. Neuron 46: 671-679, 2005.

Deco G, Lee TS. The role of early visual cortex in visual integration: a neural model of recurrent interaction. Eur J Neurosci 20: 1089-1100, 2004.

Deco G, Rolls ET. A neurodynamical cortical model of visual attention and invariant object recognition. Vision Res 44: 621-644, 2004.

Deco G, Rolls ET. Attention, short-term memory, and action selection: a unifying theory. Prog Neurobiol 76: 236-256, 2005a.

Deco G, Rolls ET. Neurodynamics of biased competition and co-operation for attention: a model with spiking neurons. J Neurophysiol 94: 295-313, $2005 b$.

Deco G, Rolls ET. Decision-making and Weber's Law: a neurophysiological model. Eur J Neurosci 24: 901-916, 2006

Deco G, Rolls ET, Horwitz B. "What" and "where" in visual working memory: a computational neurodynamical perspective for integrating fMRI and single-neuron data. J Cogn Neurosci 16: 683-701, 2004.

Desimone R, Duncan J. Neural mechanisms of selective visual attention. Aпnи Rev Neurosci 18: 193-222, 1995.

Douglas RJ, Markram H, Martin KAC. Neocortex. In: The Synaptic Organization of the Brain, edited by Shepherd GM. Oxford, UK: Oxford Univ. Press, 2004, p. 499-558.

Downar J, Crawley AP, Mikulis DJ, Davis KD. A multimodal cortical network for the detection of changes in the sensory environment. Nat Neurosci 3: 277-283, 2000.

Friston KJ, Buechel C, Fink GR, Morris J, Rolls ET, Dolan RJ. Psychophysiological and modulatory interactions in neuroimaging. NeuroImage 6: 218-229, 1997.

Friston KJ, Glaser DE, Henson RN, Kiebel S, Phillips C, Ashburner J. Classical and Bayesian inference in neuroimaging: applications. NeuroImage 16: 484-512, 2002.

Genovese CR, Lazar NA, Nichols T. Thresholding of statistical maps in functional neuroimaging using the false discovery rate. NeuroImage 15: $870-878,2002$

Gitelman DR, Penny WD, Ashburner J, Friston KJ. Modeling regional and psychophysiologic interactions in fMRI: the importance of hemodynamic deconvolution. NeuroImage 19: 200-207, 2003.

Gottfried JA, O'Doherty J, Dolan RJ. Appetitive and aversive olfactory learning in humans studied using event-related functional magnetic resonance imaging. J Neurosci 22: 10829-10837, 2002.

Gottfried JA, Winston JS, Dolan RJ. Dissociable codes of odor quality and odorant structure in human piriform cortex. Neuron 49: 467-479, 2006.

Grabenhorst F, Rolls ET. Selective attention to affective value alters how the brain processes taste stimuli. Eur J Neurosci 27: 723-729, 2008.

Grabenhorst F, Rolls ET. Different representations of relative and absolute value in the human brain. NeuroImage 48: 258-268, 2009.

Grabenhorst F, Rolls ET, Bilderbeck A. How cognition modulates affective responses to taste and flavor: top down influences on the orbitofrontal and pregenual cingulate cortices. Cereb Cortex 18: 1549-1559, 2008a.
Grabenhorst F, Rolls ET, Margot C, da Silva MAAP, Velazco MI. How pleasant and unpleasant stimuli combine in different brain regions: odor mixtures. J Neurosci 27: 13532-13540, 2007.

Grabenhorst F, Rolls ET, Parris BA. From affective value to decisionmaking in the prefrontal cortex. Eur J Neurosci 28: 1930-1939, 2008b.

Grabenhorst F, Rolls ET, Parris BA, D'Souza A. How the brain represents the reward value of fat in the mouth. Cereb Cortex 20: 1082-1091, 2009.

Haase L, Cerf-Ducastel B, Murphy C. Cortical activation in response to pure taste stimuli during the physiological states of hunger and satiety. NeuroImage 44: 1008-1021, 2009.

Henson R. Analysis of fMRI time series. In: Human Brain Function (2nd ed.), edited by Friston KJ, Zeki S, Frith CD, Dolan RJ, Price CJ. San Diego, CA: Elsevier/Academic Press, 2004, p. 793-822.

Jones EG, Powell TPS. An anatomical study of converging sensory pathways within the cerebral cortex of the monkey. Brain 93: 793-820, 1970.

Kable JW, Glimcher PW. The neural correlates of subjective value during intertemporal choice. Nat Neurosci 10: 1625-1633, 2007.

Kanwisher N, Wojciulik E. Visual attention: insights from brain imaging. Nat $\operatorname{Rev}$ 1: 91-100, 2000.

Kouneiher F, Charron S, Koechlin E. Motivation and cognitive control in the human prefrontal cortex. Nat Neurosci 12: 939-945, 2009.

Kringelbach ML, O'Doherty J, Rolls ET, Andrews C. Activation of the human orbitofrontal cortex to a liquid food stimulus is correlated with its subjective pleasantness. Cereb Cortex 13: 1064-1071, 2003.

McCabe C, Rolls ET. Umami: a delicious flavor formed by convergence of taste and olfactory pathways in the human brain. Eur J Neurosci 25: 1855-1864, 2007.

Nitschke JB, Dixon GE, Sarinopoulos I, Short SJ, Cohen JD, Smith EE, Kosslyn SM, Rose RM, Davidson RJ. Altering expectancy dampens neural response to aversive taste in primary taste cortex. Nat Neurosci 9: 435-442, 2006.

O'Doherty J, Rolls ET, Francis S, Bowtell R, McGlone F. The representation of pleasant and aversive taste in the human brain. J Neurophysiol 85: 1315-1321, 2001.

O'Doherty JP, Buchanan TW, Seymour B, Dolan RJ. Predictive neural coding of reward preference involves dissociable responses in human ventral midbrain and ventral striatum. Neuron 49: 157-166, 2006.

O'Doherty JP, Dayan P, Friston K, Critchley H, Dolan RJ. Temporal difference models and reward-related learning in the human brain. Neuron 38: 329-337, 2003.

Oram MW, Foldiak P, Perrett DI, Sengpiel F. The "Ideal Homunculus": decoding neural population signals. Trends Neurosci 21: 259-265, 1998.

Pessoa L. How do emotion and motivation direct executive control? Trends Cogn Sci 13: 160-166, 2009.

Plassmann H, O'Doherty J, Rangel A. Orbitofrontal cortex encodes willingness to pay in everyday economic transactions. J Neurosci 27: 9984-9988, 2007

Price JL. Connections of orbital cortex. In: The Orbitofrontal Cortex, edited by Zald DH, Rauch SL. Oxford, UK: Oxford Univ. Press, 2006, p. 39-55.

Pritchard TC, Hamilton RB, Morse JR, Norgren R. Projections of thalamic gustatory and lingual areas in the monkey, Macaca fascicularis. J Comp Neurol 244: 213-228, 1986.

Rolls ET. Central nervous mechanisms related to feeding and appetite. $\mathrm{Br} \mathrm{Med}$ Bull 37: 131-134, 1981

Rolls ET. Emotion Explained. Oxford, UK: Oxford Univ. Press, 2005.

Rolls ET. Sensory processing in the brain related to the control of food intake. Proc Nutr Soc 66: 96-112, 2007a.

Rolls ET. Understanding the mechanisms of food intake and obesity. Obes Rev 8: 67-72, 2007b.

Rolls ET. Functions of the orbitofrontal and pregenual cingulate cortex in taste, olfaction, appetite and emotion. Acta Physiol Hung 95: 131-164, 2008a.

Rolls ET. Memory, Attention, and Decision-Making: A Unifying Computational Neuroscience Approach. Oxford, UK: Oxford Univ. Press, 2008b.

Rolls ET. Taste, olfactory and food texture processing in the brain and the control of appetite. In: Obesity Prevention, edited by Dube L, Bechara A, Dagher A, Drewnowski A, LeBel J, James P, Yada RY. London: Elsevier/ Academic Press, 2010, p. 41-56.

Rolls ET, Deco G. Computational Neuroscience of Vision. Oxford, UK: Oxford Univ. Press, 2002.

Rolls ET, Deco G. The Noisy Brain: Stochastic Dynamics as a Principle of Brain Function. Oxford, UK: Oxford Univ. Press, 2010.

Rolls ET, Franco L, Aggelopoulos NC, Reece S. An information theoretic approach to the contributions of the firing rates and correlations between the firing of neurons. J Neurophysiol 89: 2810-2822, $2003 \mathrm{a}$. 
Rolls ET, Grabenhorst F. The orbitofrontal cortex and beyond: from affect to decision-making. Prog Neurobiol 86: 216-244, 2008.

Rolls ET, Grabenhorst F, Margot C, da Silva MAAP, Velazco MI. Selective attention to affective value alters how the brain processes olfactory stimuli. J Cogn Neurosci 20: 1815-1826, 2008a.

Rolls ET, Grabenhorst F, Parris BA. Neural systems underlying decisions about affective odors. J Cogn Neurosci 22: 1069-1082, 2010.

Rolls ET, Kringelbach ML, de Araujo IET. Different representations of pleasant and unpleasant odors in the human brain. Eur J Neurosci 18: 695-703, 2003b.

Rolls ET, McCabe C, Redoute J. Expected value, reward outcome, and temporal difference error representations in a probabilistic decision task. Cereb Cortex 18: 652-663, 2008b.

Rolls ET, Rolls BJ, Rowe EA. Sensory-specific and motivation-specific satiety for the sight and taste of food and water in man. Physiol Behav 30: 185-192, 1983.

Rossi AF, Pessoa L, Desimone R, Ungerleider LG. The prefrontal cortex and the executive control of attention. Exp Brain Res 192: 489-497, 2009.

Sakai K, Passingham RE. Prefrontal interactions reflect future task operations. Nat Neurosci 6: 75-81, 2003.

Sakai K, Passingham RE. Prefrontal set activity predicts rule-specific neural processing during subsequent cognitive performance. J Neurosci 26: 12111218, 2006.
Schoenfeld MA, Neuer G, Tempelmann C, Schussler K, Noesselt T, Hopf JM, Heinze HJ. Functional magnetic resonance tomography correlates of taste perception in the human primary taste cortex. Neuroscience 127: 347-353, 2004.

Small DM, Gregory MD, Mak YE, Gitelman D, Mesulam MM, Parrish T. Dissociation of neural representation of intensity and affective valuation in human gustation. Neuron 39: 701-711, 2003.

Veldhuizen MG, Bender G, Constable RT, Small DM. Trying to detect taste in a tasteless solution: modulation of early gustatory cortex by attention to taste. Chem Senses 32: 569-581, 2007.

Wang XJ. Probabilistic decision making by slow reverberation in cortical circuits. Neuron 36: 955-968, 2002.

Wang XJ. Decision making in recurrent neuronal circuits. Neuron 60: 215234, 2008.

Wilson JL, Jenkinson M, Araujo IET, Kringelbach ML, Rolls ET, Jezzard P. Fast, fully automated global and local magnetic field optimisation for fMRI of the human brain. NeuroImage 17: 967-976, 2002.

Yacubian J, Glascher J, Schroeder K, Sommer T, Braus DF, Buchel C. Dissociable systems for gain- and loss-related value predictions and errors of prediction in the human brain. J Neurosci 26: 9530-9537, 2006.

Yaxley S, Rolls ET, Sienkiewicz ZJ. Gustatory responses of single neurons in the insula of the macaque monkey. J Neurophysiol 63: 689-700, 1990. 